\title{
MOLAR INMADURO DE MAMUT EN GRAN DOLINA (ATAPUERCA, BURGOS)
}

\author{
Emiliano AGUIRRE
}

Museo Nacional de Ciencias Naturales, CSIC. 28006 Madrid (España)

\begin{abstract}
Aguirre, E. 1999. Molar inmaduro de mamut en Gran Dolina (Atapuerca, Burgos). [Mammoth milk molar in Gran Dolina (Atapuerca, Burgos)]. Revista Española de Paleontología, n" extr. Homenaje al Prof. J. Truyols, 135-142. ISSN 0213-6937.
\end{abstract}

\begin{abstract}
An inmature elephant tooth was recovered from a fallen block of the TD6 Beds, in the Atapuerca Railway Cut, at the foot of Gran Dolina section. Such dental pieces are seldom collected, their diagnostic traits are feeble, and are but rarely illustrated in the literature. The tooth is not fully preserved; it was at death in a state of advanced abrasion. The observable and comparable numeric traits allow its identification as a last lower milk molar, D4, of the genus Mammuthus. The piece is assigned, not with full security, to a progressive variety of M. meridionalis —either M. m. "tamanensis", or M. m. "voigstedtensis", or "cromerensis" or similar- more probably than to a primitive variety of $M$. trogontherii.
\end{abstract}

Keywords: Atapuerca, Gran Dolina, mammoth, Mammuthus meridionalis, Mammuthus trogontherii, Pleistocene.

\section{RESUMEN}

Un diente yugal de un elefántido inmaduro fue recogido de un bloque desprendido de las capas TD6, caído al fondo de la Trinchera de Atapuerca. Estas piezas son raras en las colecciones; en ellas los rasgos diagnósticos son débiles, y son muy escasas las referencias en la literatura. La pieza no está del todo completa, y se halla en un estado de desgaste avanzado. Se identifica como un último molar de leche, D4, inferior, del género Mammuthus, y se atribuye con alguna duda a una subespecie progresiva de $M$. meridionalis $-M$. m. "tamanensis", $M$. m. "voigstedtensis", M. m. "cromerensis", etc.—, más bien que a un primitivo M. trogontherii.

Palabras clave: Atapuerca, Gran Dolina, mamut, Mammuthus meridionalis, Mammuthus trogontherii, Pleistoceno.

\section{INTRODUCCIÓN}

En 1985 se desprendieron y cayeron unos bloques del corte de depósitos en la Gran Dolina de la Trinchera de Atapuerca (Aguirre, 1995; Hoyos y Aguirre, 1995). Por la cicatriz fresca que quedó en el corte y la naturaleza de los materiales sedimentarios, se conoce con seguridad y sin lugar a dudas el tramo de la sección estratigráfica al que cabe atribuir un número de fósiles que fueron recogidos en el fondo de la trinchera, a poco de ocurrir el derrumbe, entre los terrones caídos, al pie del corte, y que corresponde a las capas TD6.

Entre los fósiles recogidos, se cuenta una pieza dentaria de proboscidio (Fig. 1), grupo sin representación en las listas faunísticas de Atapuerca, hasta entonces. Se la citó como especie indeterminada, Mammuthus sp. cf. trogontherii. La identificación de la especie representada por esta sola pieza es sencillamente difícil. Son muy escasas las referencias de muelas inmaduras de elefántidos en la literatura. Entre varios miles de dientes yugales de elefántidos estudiados en docenas de colecciones de todo el mundo para la tesis doctoral del firmante son raros los D4 y no se halla ningún D4 inferior del mamut meridional. Aun cuando el resultado de su estudio no es brillante, tampoco carece de interés y es bueno contribuir con él al homenaje que esta Sociedad y Revista de Paleontología dedican al gran amigo, compañero y maestro que es Jaime Truyols.

La singular dinámica ontogénica del aparato dental de los elefantes es causa de dificultades peculiares en el estudio de sus fósiles. Cada diente es distinto del otro en todas sus dimensiones. No sólo ha de compararse cada pieza dentaria con su homóloga, sino que la seguridad en los resultados del estudio biométrico comparativo es 


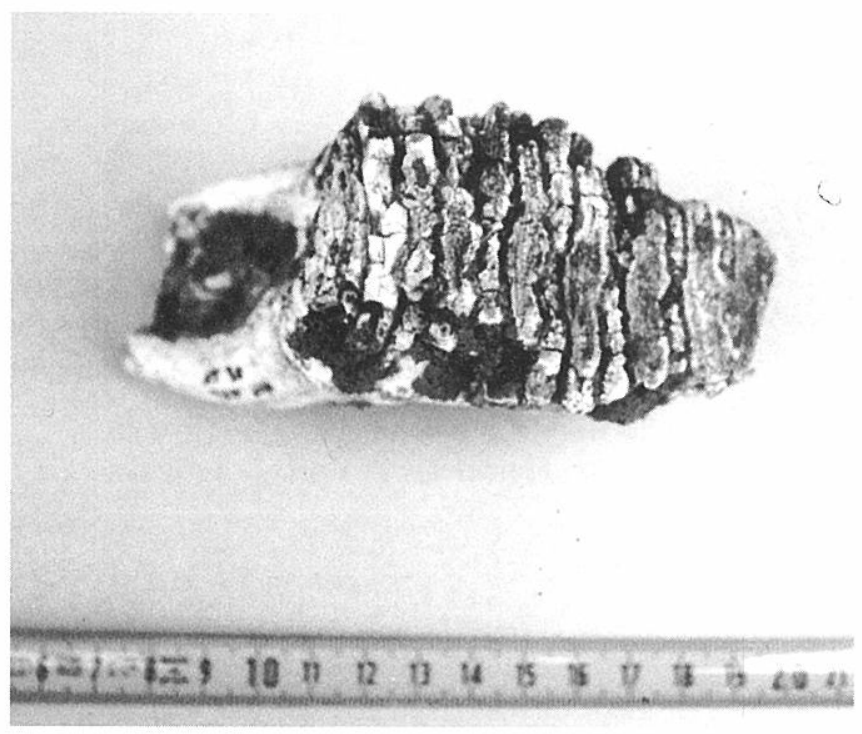

Figura 1. Diente inmaduro de Mammuthus sp., del nivel TD6 en la Trinchera de Atapuerca.

notablemente menor si se toman piezas homólogas en cualquier situación de su desgaste —inicial, medio o final - que si se comparan sólo piezas homólogas y con desgaste similar. En piezas muy gastadas no cabe medir el largo ni el ancho ni la altura simples o totales de la pieza. El recurso de tomar como variables funcionales las que pueden contarse en la superficie oclusal, limitada a las láminas afectadas por el desgaste (Aguirre, 1968), integrada en casos por parte de dos dientes yugales en relevo, ayuda a resolver, pero también afecta a las variables numéricas, como recordaremos más adelante, con un gradiente según la edad del individuo y la evolución progresiva en cada linaje.

\section{DESCRIPCIÓN DE LA PIEZA DENTARIA}

En el caso de Atapuerca, se trata de un diente muy desgastado, no extremadamente: las láminas anteriores lo están hasta la base, o casi, y las posteriores hasta cerca de la mitad. La erupción de la pieza siguiente estaba, inferencialmente, avanzada, y no es improbable que hubieran comenzado a desgastarse los ápices de hasta dos o tres láminas de esta última. Se conserva la base del diente, no el ecuador de la corona que es normalmente más largo. No disponemos, pues, de medidas del largo ni de la altura totales de la pieza. La raíz posterior se conserva casi entera, la anterior falta por completo, salvo la parte próxima a la corona en su extremo anterior, donde se aprecia conservado el borde mesial de la base de la corona. La conservación de la corona es algo deficiente: falta cemento y se ha perdido parte de las láminas posteriores en un lado. No sin dudas, se puede identificar la pieza como de la serie inferior y del lado izquierdo: las diferencias entre inferiores y superiores son en los dientes lacteales mucho más tenues que en los adultos. El esmalte presenta pliegues y una expansión central de las láminas, a media altura de éstas.
La pieza dentaria de elefante de Gran Dolina es de pequeñas dimensiones, de individuo inmaduro. A primera vista podría tratarse de un último diente lacteal, D4, o del primero adulto, M1.

\section{Variables numéricas}

Hay un gradiente notable en las variables numéricas de los dientes yugales de elefantes, entre el primero y último lacteales, D2 a D4, y entre el primero y último adultos, M1 a M3; pero algunas diferencias son tenues entre el último de aquellos y el primero de éstos, lo que dificulta el diagnóstico diferencial. El D4 alcanza una longitud semejante a la del M1 y un número de láminas de esmalte muy aproximado o igual. En altura y anchura de la corona, el M1 está muy por debajo del M2; con todo, excede sensiblemente en estas dimensiones y en corpulencia al D4 en un mismo individuo. En la raíz posterior del D4 convergen cavidades pulpares de varias láminas, y puede ser robusta, asemejándose a la del M1.

La longitud funcional (LF) - longitud del área desgastada de la muela o muelas actualmente en función (Aguirre, 1968-1969)- medida, 81,2 mm, puede no haber sido la real, si, como se indicaba más arriba, había comenzado el uso y desgaste de la pieza siguiente.

$\mathrm{La}$ anchura funcional (AF) medida por el ancho mayor de lámina afectado por el desgaste, 49,55 mm, es próxima a la real; se ha medido algo por debajo de la actual superficie oclusal, a causa de los bordes rotos.

Una aproximación a la anchura máxima (AM) es posible, pues nunca sería menor que el ancho máximo de la lámina más ancha. Las láminas posteriores parecen haber tenido la anchura mayor en la parte conservada, esto es, en la mitad inferior de la corona dentaria. Es posible colegir que el ancho mayor de la muela de TD6 podría acercarse o superar apenas los $50 \mathrm{~mm}$ medidos en la lámina más ancha.

Se han contado 9 láminas masticadoras —los guantes de esmalte transversales y paralelos, que resultan de la fusión de cúspides alineadas, en los dientes yugales de elefántidos-, incluyendo las láminas poco desarrolladas o incompletas que los autores llaman "talones", y que pueden apreciarse al menos por una débil pared de esmalte conservada. En correspondencia con la longitud funcional medida, el número de láminas actualmente en uso (U) es de ocho.

Es válido el índice de frecuencia laminar funcional (IQ) — según definición de Aguirre (1968-1969)— que se obtiene, en este caso, dividiendo el número de láminas en oclusión observado (U) por la longitud funcional medida (LF). Este índice varía ligeramente a lo largo del desgaste de una misma pieza dentaria, y cuando en la superficie masticadora actual entran láminas posteriores de una pieza y las anteriores de la siguiente, en relevo. En este último caso, crece algo la longitud funcional total mientras decrece la anchura, pero aumenta también el número de láminas comprometidas en la función, pues los "talones" son más delgados y prietos. En un caso como el de Atapuerca TD6, el índice obtenido no variaría mucho respecto del que hubiera podido hallarse en un desgaste 


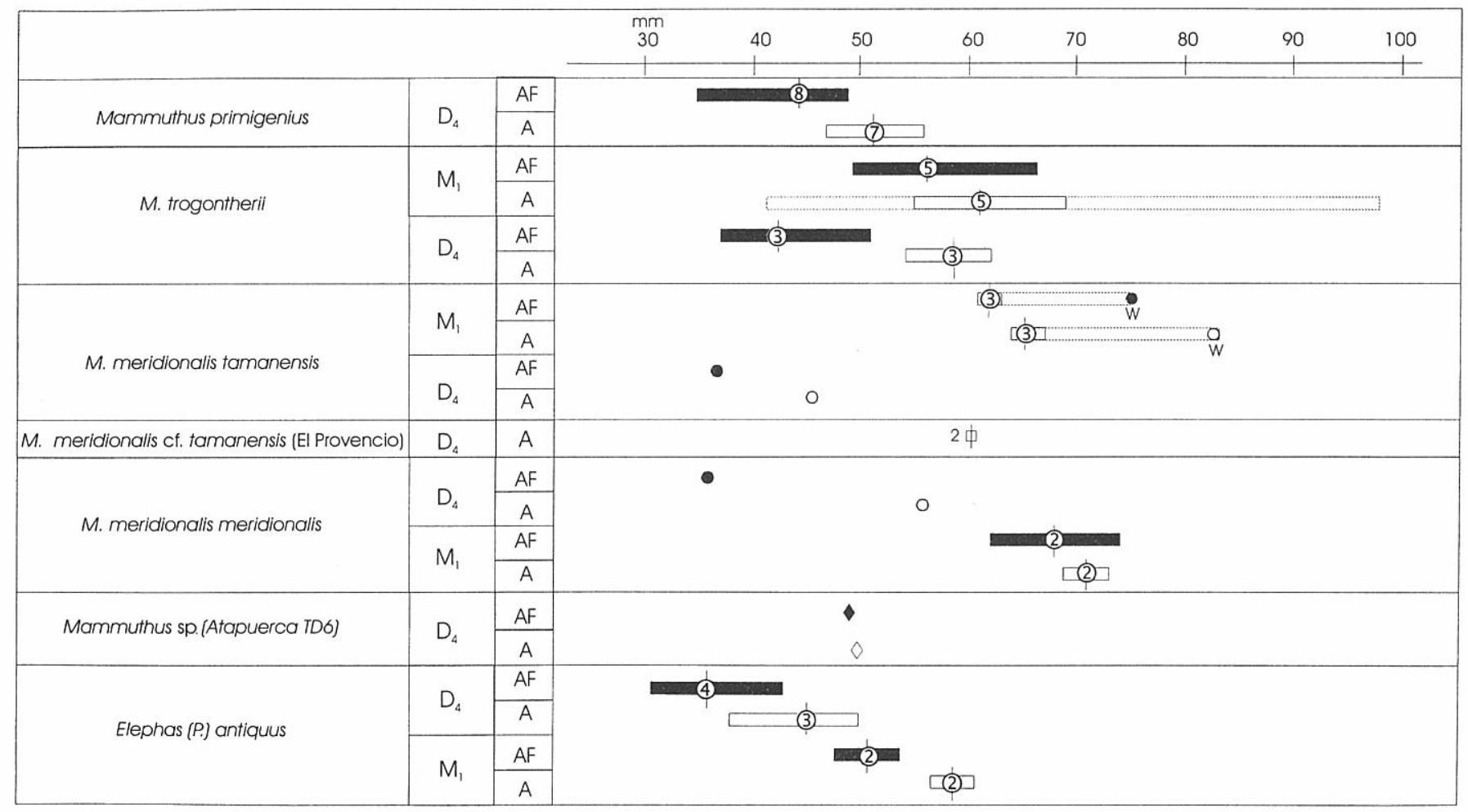

Figura 2. Anchuras, máxima (A) y funcional (AF), de molares inmaduros —último lacteal (D4) y primero adulto (M1) inferiores de elefántidos fósiles y del diente de mamut de Atapuerca TD6 $(\diamond, \diamond)$.

Nota: el número sobre la barra indicadora del recorrido representa el tamaño de la muestra $(\mathrm{N})$, y está centrado sobre el valor de la media (x), w: valores de un ejemplar atribuido a Elephas "wüsti". Barras y signos para la magnitud funcional.

mediano de una pieza igual, ni tampoco respecto del que hubieran dado las dos piezas en relevo hipotético. El índice de anchura funcional sí está más alejado del que se hubiera obtenido en esta segunda hipótesis, y algo también del que tendría la pieza sola con desgaste menos avanzado.

El grosor medido del esmalte(EX), como media estadística (ver Aguirre 1968-1969), es de 1.95 mm. Poco material hay de comparación, pues en mis trabajos anteriores no atendía a esta variable sino en molares adultos (MM2, MM3), y en la bibliografía las referencias son escasas (ver más abajo).

\section{CUESTIÓN TAXONÓMICA}

El estudio comparativo ha de hacerse con los elefántidos fósiles conocidos de Europa, del género Elephas Linné, E. (Palaeoloxodon) antiquus Falconer, y del género Mammuthus (Brooken). De este último se reconocen en Europa cuatro especies: la especie tipo, $M$. primigenius Blumenbach, que es la más moderna, de finales del Pleistoceno Medio al Holoceno; la más primitiva reconocida hasta ahora, $M$. gromovi Garutt y Alexeeva, que sólo se conoce en el Plioceno; sigue $M$. meridionalis Nesti, en el Plioceno Superior, todo el Pleistoceno Inferior y primera parte del Pleistoceno Medio; en la mayor parte del Pleistoceno Medio, el género Mammuthus está representado por el mamut de estepa, $M$. trogontherii Pohlig: este sinónimo ha prevalecido por el uso, contra la ley de prioridad, sobre el original M. armeniacus Falconer (Aguirre, 1969).

Las sucesivas especies de Mammuthus son anagenéticas. Se caracterizan por el progreso evolutivo en ciertas líneas: corona hipsodonta de los molares, aumento del número - absoluto y relativo- de láminas masticadoras, adelgazamiento del esmalte, posición más alta de la anchura máxima en la corona. Esta historia de progreso evolutivo tiene fases distintas, en un número mayor que el de especies: así, en el mamut de estepa se distinguen una subespecie típica $-M$. t. trogontherii- y otras progresivas, M. t. leithadamsi Pohlig y M. t. fraasi Jourdan. De modo semejante se fueron dando nombres subespecíficos a ejemplares o colecciones de fósiles que se clasificaban como mamut meridional, pero connotando su carácter progresivo en la tendencia hipsodonta y las otras relacionadas: $M$. meridionalis cromerensis Depéret y Mayet para fósiles de Francia, hoy en desuso; $M$. $m$. voigstedtensis Dietrich en Europa central; $M . m$. tamanensis Dubrovo para el sudeste de Europa; $M . m$. vestinus Azzaroli en Italia. Todos ellos se datan entre poco antes del episodio paleomagnético Jaramillo y ya bien empezado el Brunhes (entre c. 1,2 y algo menos de $0,7 \mathrm{Ma}$ ), y se distinguen del tipo de mamut meridional por las mismas características aproximadamente. Hasta hoy no se ha discutido si constituyen subespecies distintas entre sí por otra cosa que la distribución 


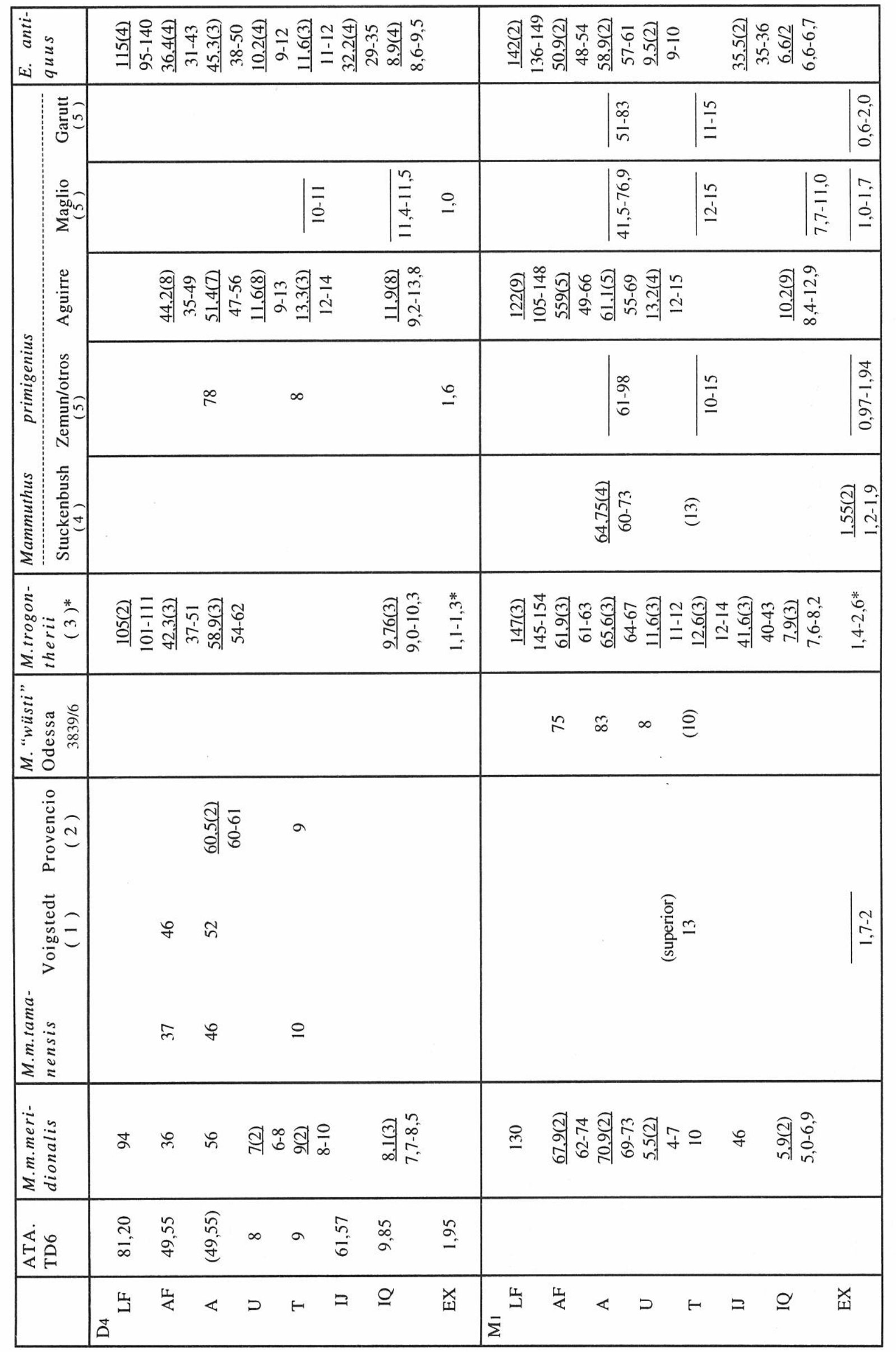

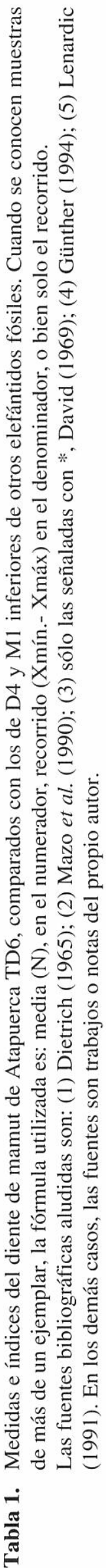




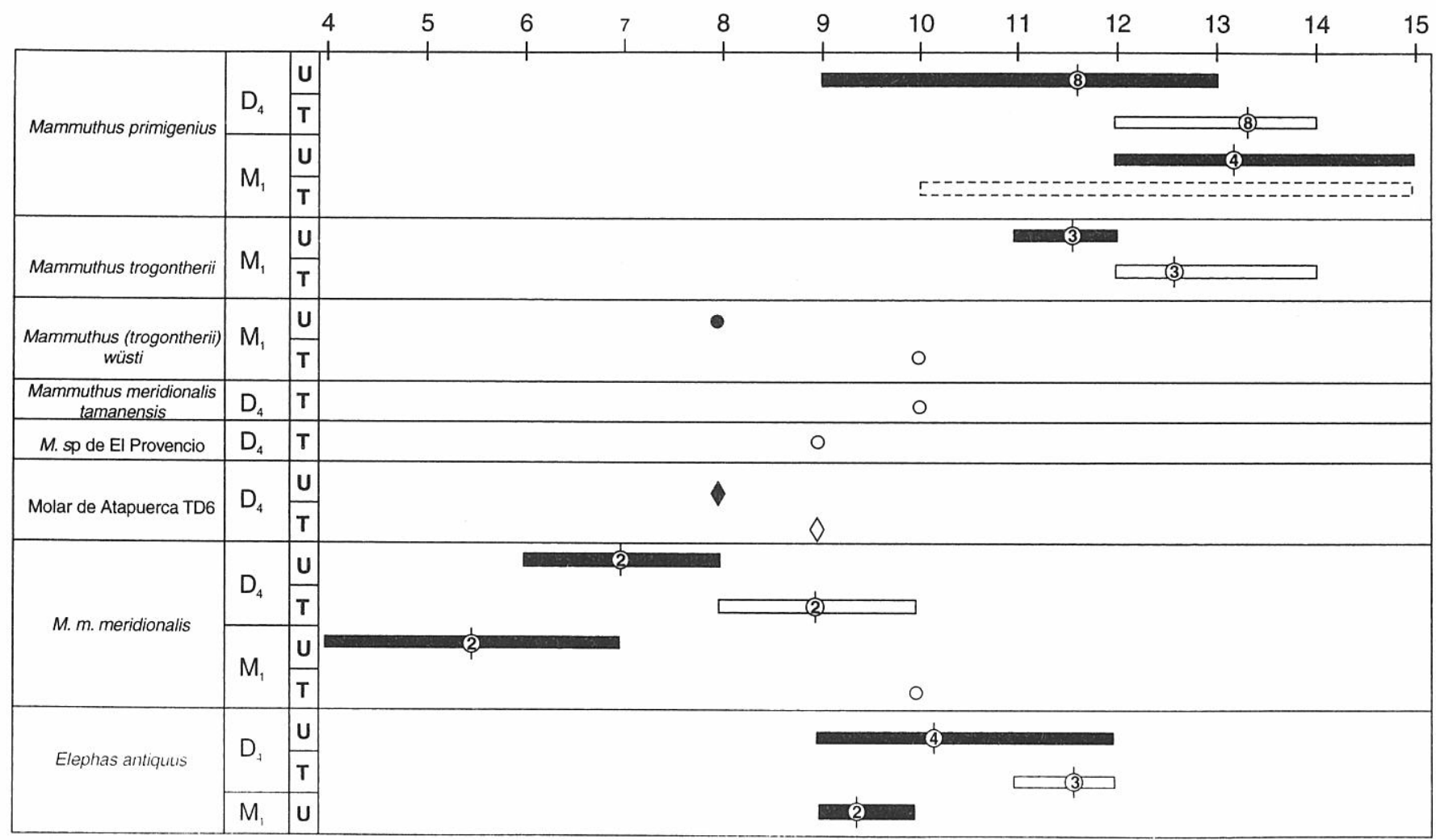

Figura 3. Número de láminas total (T) y funcional (U) en el último molar lacteal (D4) y primer molar adulto (M1) inferiores de elefántidos fósiles de Europa y el de Atapuerca TD6 $(\diamond, \diamond)$.

Nota: signos como en la Fig. 2. Barra de trazos, según datos de bibliografía.

geográfica; de modo que sí se distinguen a nivel subespecífico del $M$. meridionalis meridionalis, pero no puede decirse si como una, dos o más subespecies, ni cuál, en su caso, tendría la prioridad. Sí convienen varios autores en señalar de un modo informal el parecido entre todas estas muestras, y se dice de todas ellas que son "close relatives" (Azzaroli et al., 1997), "forma evolucionada", "forma progesiva" o "forma final filética de la macrospecie ("Grossart") E. meridionalis" (Dietrich, 1965). En el caso de España cabe, o crear un nuevo nombre subespecífico geográfico, o escoger por no sé qué criterios uno de los otros, o desistir de designarlos por un nombre taxonómico formal. Prefiero esto último, y dejarlo abierto como "subespecie progresiva" sin nombre, o como una de las "variedades progresivas", dejando a un lado la cuestión de la categoría taxonómica.

No está de más recordar una pequeña dificultad añadida, que es el caos nomenclatural que afecta a los dientes yugales inmaduros de elefantes. Los dientes lacteales en esta familia, desde muy cerca del inicio de su evolución, no son reemplazados por muelas permanentes en posición homóloga, sino que el hueso se reabsorbe a lo largo de su progreso y tras su caída, de modo que sólo los sustituye el primer molar adulto, M1. La última muela de leche se designa, según los autores, D4, DP4, dm4 o dm3, dd3, mm3, Pd4, dP5 o MM1.

\section{ESTUDIO COMPARATIVO}

El material de comparación al que nos vamos a referir incluye piezas estudiadas y medidas directamente por el propio firmante, y otras conocidas por la literatura. Las primeras son:

- Un D4 inferior, poco gastado, de M. meridionalis típico: de Oscuragnolo, Valdarno (colección antigua).

- D3+D4 inferiores en relevo; M. meridionalis tamanensis: de Ukrania, Pleistoceno Inferior.

- Dos D4 inferiores, uno muy gastado, el otro poco; $M$. trogontherii: de Süssenborn, Complejo Cromeriense, Pleistoceno Medio bajo.

- Un D4 inferior, aislado, de $M$. trogontherii: de Mosbach superior, Pleistoceno Medio.

- Dos D4 inferiores, en relevo con D3, izquierdo y derecho, del mismo individuo, de $M$. meridionalis progresivo: de El Provencio, atribuida a final del Plesitoceno Inferior (Mazo et al., 1990).

- D3+D4 inferiores en relevo; M. trogontherii: de CúllarBaza, Pleistoceno Medio (v. Mazo, 1989).

- Una muestra, con N=4, de D4 inferiores de Elephas antiquus de varias procedencias.

- Una muestra, con ocho D4 inferiores de M. primigenius de varias procedencias.

- Muestra de dos MM1 inferiores de M. meridionalis típicos; varias procedencias. Solos $(s)$ y muy gastados $(r)$. 


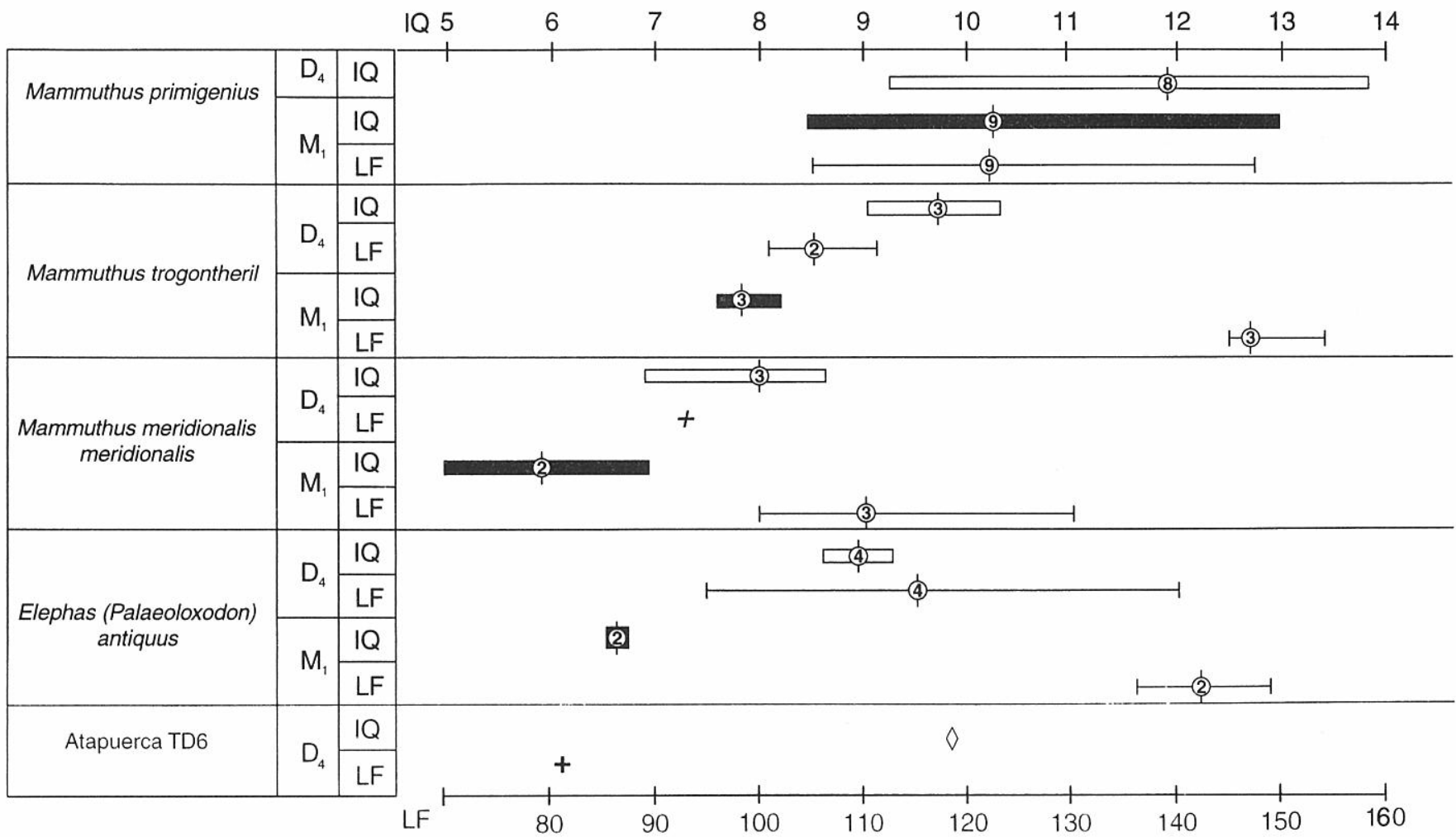

Figura 4. Índice de frecuencia laminar funcional (IQ) y longitud funcional (LF) en el diente de mamut de Atapuerca TD6 $(\diamond+)$, y otros elefántidos de Europa; última muela de leche (D4) y primera adulta inferiores.

Nota: Barras para el índice IQ: blancas para D4, negras para M1; líneas para las longitudes funcionales (LF). Lo demás como en las Figs. 2 y 3.

- Muestra de tres MM1 inferiores de M. trogontherii; varias procedencias: $s$ y $r$.

- Muestra de cinco MM1 inferiores de M. primigenius; varias procedencias: $s$ y $r$.

- Muestra de dos MM1 inferiores de E. antiquus; varias procedencias: $s$ y $r$.

- Un M1 inferior de "Elephas wüsti" (M. trongontherii típico según Dubrovo, 1975) de Tiraspol (procedencia no del todo confirmada), en el Museo Paleontológico de Odessa, con referencia 3839/6.

En la literatura tampoco es fácil encontrar datos numéricos ni estudios de piezas dentarias juveniles o inmaduras de elefantes fósiles. Para el presente trabajo se ha contado con datos de un D4 superior y un M1 también superior de Mammuthus meridionalis voigstedtensis de Dietrich (1965), y un probable M1 de M. primigenius de Stuckenbush (Günther, 1994); Lenardic (1991) refiere asimismo datos de M. primigenius según Maglio y Garutt, junto con los obtenidos por la misma autora para algunos ejemplares aislados de diversas procedencias. De una muestra de la terraza Kolkotova del Dniester, Moldavia, y otros afloramientos correlacionados, ofrece varias medidas David (1969). Este autor refiere a Mammuthus trogontherii Pohlig típico — como hará más tarde Dubrovó (1975)— la muestra de las mismas localidades conocida desde antiguo y clasificada primero como "Elephas wüsti" (Pavlova), y más tarde por la misma autora como "Elephas trogontherii var. meridionalis" (Pavlova). Los datos del presente estudio comparativo se resumen en la Tabla 1.

\section{Discusión}

La anchura está por debajo de las conocidas para M1, salvo algún caso anómalo de los que se presentan en el mamut lanudo, M. primigenius. El índice laminar funcional, aun cuando algo sesgado, como se ha dicho, queda también muy por encima de los M1 de $M$. meridionalis y $M$. trogontherii conocidos, y más próximo a los de sus D4. Queda, pues, poco lugar a dudas de que se trata de un D4, última muela de leche, inferior (Fig. 2).

En cuanto a la asignación taxonómica, los números de láminas, total y en ejercicio, son del orden de los registrados en los ejemplares de D4, y también de los M1 clasificados como variedad progresiva de $M$. meridionalis - M. m. tamanensis, M. m. voigstedtensis, entre otros nombres-, y en uno atribuido a "Elephas wüsti", que se identifica como $M$. trogontherii primitivo o típico (Dubrovo, 1975), y están lejos tanto de M. meridonalis meridionalis (o típico) como de M. primigenius. Estos valores son bajos también para los observados en D4 de E. antiquus. El índice laminar funcional (IQ, Tabla 1) (definido en Aguirre, 1968-1969) es en el diente de Gran Dolina próximo a la media de los D4 de $M$. trogontherii, superior a las muestras conocidas de $M . m$. meridionalis $s_{-}$y bajo para $M$. primigenius. Es una tendencia progresiva en la evolución del género. Las anchuras de la pieza en estudio entran en los márgenes de variación de los D4 de las especies del género Mammuthus, y exceden las de Elephas antiquus. Las figuras de las secciones laminares 
son diferentes en uno y otro género: en el caso estudiado corresponden al patrón de los mamut. Con seguridad, pues, se asigna el fósil al género Mammuthus con exclusión de Elephas (o Palaeoloxodon).

Al no poder medirse la altura, carecemos también de valores de hipsodoncia, un factor claramente en aumento a lo largo de la evolución del género Mammuthus (Aguirre, 1969, 1972). El número de láminas, el total y las que se cuentan funcionando a la vez en la superficie oclusal, son variables claramente progresivas en la evolución de este género, desde el inicio del episodio magnético Jaramillo hasta su extinción al final del Pleistoceno (Figs. 3 y 4). En resumen, por los números laminares, el fósil en estudio parece estar más cerca de los mamuts meridionales progresivos (M. m. tamanensis) que de los mamuts de estepa ( $M$. trogontherii); a éstos parece acercarle más la frecuencia laminar —que puede ser sesgada- Con no ser decisivas las anchuras, puede tener peso una apreciación indirecta de la hipsodoncia: en el fósil de TD6 la anchura máxima parece registrarse en la parte inferior y próxima a la base de la corona, como es común en $M$. meridionalis, incluso en algunas variedades progresivas, como la de Voigstedt (Aguirre, 1972), y al contrario que en $M$. trogontherii, donde la mayor anchura se halla más próxima a la media altura de la corona. Esta diferencia es muy clara y diagnóstica en molares adultos, y puede no ser decisiva en los de leche, donde los rasgos más progresivos en la evolución de un grupo son normalmente menos manifiestos.

En cuanto al grosor medio de las capas de esmalte, que crece en la serie molar con la edad del individuo, pero es decreciente en la evolución del género Mammuthus (Aguirre, 1968-1969) y varía moderadamente entre láminas centrales y distales de una pieza, el valor hallado para el ejemplar de TD6 (1,95 $\mathrm{mm})$ es ligeramente superior al medido en una pieza homóloga sólo empezada a desgastar de $M$. trogontherii de CúllarBaza (Mazo, 1989), está dentro de los límites señalados para M1 superiores de $M$. meridionalis voigstedtensis por Dietrich (1965) y para M1 inferiores de $M$. trogontherii del Dniester, nivel de aluvión superior de Kolkotova, por David (1969); y está fuera por encima de todo lo citado para D4 y M1 inferiores de $M$. primigenius (Maglio, 1973, y Garutt, 1976, fide Lenardic, 1991; Günther, 1994). Converge, pues, también éste con otros criterios en señalar proximidad con los meridionales progresivos y los de estepa primitivos: la continuidad evolutiva de aquellos a éstos se tiene por segura, y hace en muchos casos difícil el diagnóstico incluso en molares adultos.

\section{CONCLUSIÓN}

Sin despejar todas las dudas, puede asignarse el diente fósil de elefántido de Atapuerca, TD6, a un último molar de leche, inferior e izquierdo, de una variedad de mamut, entre el Mammuthus meridionalis evolucionado y el $M$. trogontherii, más probablemente identificable con una de las variedades o subespecies progresivas del mamut meridional. Estas últimas aparecen en Pirro e Imola, se conocen en yacimientos con signo magnético del Jaramillo en Europa Oriental, y algo más tarde en Voigstedt y Gombasek, ya en el Brunhes; en España, en Loma de Taberneros de Fuensanta (Mazo et al., 1990). Los primeros $M$. trogontherii propiamente dichos son de Tiraspol y Süssenborn, todavía con faunas de Mimomys savini, anteriores a las de Arvicola cantiana.

\section{AGRADECIMIENTOS}

El autor agradece las ayudas al Proyecto de Atapuerca, de la CICYT, la asistencia del Grupo Espeleológico Edelweiss de Burgos, y en especial a Ana Mazo, que dedicó tiempo a la búsqueda de datos y criterios para este estudio.

\section{BIBLIOGRAFÍA}

Aguirre, E. 1968-69. Revisión sistemática de los Elephantidae por su morfología y morfometría dentaria. Estudios Geológicos, 24, 109-167; 25, 123-177; 317367.

Aguirre, E. 1969. Evolutionary History of the Elephant. Science, 164, 1366-1376.

Aguirre, E. 1972. Utilization of proboscidians in Pleistocene stratigraphy. In: International Colloquium on the problem "The Boundary between Neogene and Quaternary” (Eds. M.N. Alekseev, E.A. Vangengeim, K.V. Nikiforova and I, M. Khoreva). Collection of Papers, vol. 3. Moscow, INQUA, 3-13.

Aguirre, E. 1995. Atapuerca (Burgos, España): su contribución a las ciencias del Cuaternario. Revista Española de Paleontología, 10, 58-82.

Azzaroli, A., Colalongo, M.L., Nakagawa, H., Pasini, G., Río, D., Ruggieri, G., Sartoni, S. and Sprovieri, R. 1977. The Pliocene-Pleistocene boundary in Italy. In: The Pleistocene Boundary and the Beginning of the Quaternary (Ed. J.D. van Couvering). Cambridge University Press, 141-155.

David, A. I. 1969. Mliekopitaiusche rannega Antropogena Moldavii [Mamíferos del Antropógeno inferior de Moldavia]. Izvestiya Akademii Nauk Moldavskoy SSR, Seria biologicheskij i jimicheskij Nauk, 2, 18-44 (en ruso).

Dietrich, W.O. 1965. Fossile Elefantenzähne von Voigstedt in Thüringen. Paläontologische Abhandlungen. A, Paläozoologie, 2(2/3), 521-535.

Dubrovó, I. 1975. Mammuthus trogontherii trogontherii (Pohlig) aus den Pleistozän von Tiraspol. Quartärpaläontologie, 1, 125-156.

Günther, E.W. 1969. Die Elefantenmolaren aus den Kiesen von Süssenborn bei Weimar. Paläontologische Abhandlungen. A. Paläozoologie, 3, 711-732.

Günther, E.W. 1994. Die Mammutfunde von Stuckenbush bei Herten. Geol. Paläont. Westfal., 28, 7-40.

Hoyos, M. y Aguirre, E. 1995. El registro paleoclimático pleistoceno en la evolución del karst de Atapuerca (Burgos): el corte de Gran Dolina. Trabajos de Prehistoria, 52, 31-45. 
Lenardic, J. 1991. Kranijalni dijelovi, mandibule i izolirani zubi Pleistocenskih slonova s raznih lokaliteta hrvatske i vojvodine. Geoloski vjesnik (Zagreb), 44, 15-29.

Mazo, A. 1989. Nuevos restos de Proboscidea (Mammalia) en la cuenca de Guadix- Baza. In: Geología y Paleontología de la cuenca de Guadix-Baza (Eds. M.T. Alberdi y F.P. Bonadonna). M.N.C.N., C.S.I.C., Madrid.
Trabajos sobre Neógeno-Cuaternario, monogr. 11, 225234.

Mazo, A., Pérez-González, A. y Aguirre, E. 1990. Las faunas pleistocenas de Fuensanta del Júcar y El Provencio y su signficado en la evolución del Cuaternario en la Llanura Manchega. Boletín Geológico y Minero, 101, 404-418.

Manuscrito recibido: 15 de enero, 1998 Manuscrito aceptado: 19 de junio, 1998 